\title{
Improving seed systems resiliency at local level through participatory approach for adaptation to climate change
}

\section{Opinion}

Year 2015 represented the culmination of an ambitious agenda known as Millennium Development Goals (MDGs) designed to improve human well-being globally and achieved the set goals like to reduce extreme poverty, child mortality, hunger etc. The MDGs in 2015 were replaced by the Sustainable Development Goals (SDGs) and the same were adopted by Global community. The soul of the SDGs is to eliminate extreme poverty, hunger, and malnutrition, and to conserve the environment with the core objective to end hunger and global poverty by 2030 . The SDGs Goal 2, with the objective to end hunger, achieve food security and improved nutrition, and promote sustainable agriculture signifies the critical role of enhancing adaptation to climate change vis-a-vis food and nutritional security. Also in 2015, the Paris Agreement which has been adopted by 195 Nations in Paris in December 2015 underlines the links between safeguarding food security and ending hunger and the impacts of climate change. According to the Paris Climate Change Agreement even if the increase in temperature is restricted to less than $20 \mathrm{C}$, adaptation support would be required for developing countries. The agreement establishes the global goal on adaptation-of enhancing adaptive capacity, strengthening resilience and reducing vulnerability to climate change-with a view to contributing to sustainable development and ensuring and adequate adaptation response in the context of the $20 \mathrm{C}$ goal. World food demand is expected to double by 2050 and this increase will result from a rise in world population from 7 billion in 2013 to 9 plus billion people as well as an increase in per capita consumption. To meet the ever increasing and diversified food demand, the production needs to be doubled from the available resources which are already under stress. Nevertheless, issues such as climate change, diminishing natural resources including biodiversity loss, and declining total factor productivity having serious concerns about food security.

The climate change events reflected in terms of increased frequencies of temperature extremes, frequent droughts, floods, and increased salinity have already started affecting agricultural production and productivity and particularly more so in marginal or low input environments which are home to more than two billion people which are highly insecure with respect to food security which in turn is always linked with seed security. Adaptation to climate variability and extremes events serves as a basis for reducing vulnerability to long term climate change. Strategies among others for increasing our adaptive capacity include development of adverse climate-resilient varieties.. One of the breeding strategies to mitigate climate changes is to improve adaptation through increased access to a number of varieties at local level with different growth durations to escape or avoid predictable occurrences of stress at critical periods. Many varieties are officially released, but few are adopted by farmers; by contrast farmers often grow varieties, which were not officially released. Even when new varieties are acceptable to farmers, their
Volume 6 Issue I - 2017

\author{
Rishi Pal Singh \\ Directorate of Seed \& Farms, India
}

Correspondence: Rishi Pal Singh, Directorate of Seed \& Farms, Birsa Agricultural University, Kanke, Ranchi-834006, Jharkhand, India Email dsfbau@rediffmail.com

Received: January 17, 2017| Published: January 27, 2017

seed is either not available or too expensive. Participatory research and participatory plant breeding (PPB) are addressing these specific questions while at the same time addressing some of the major global problems such as climate changes, biodiversity, and hunger. Nevertheless, the development, deployment and dissemination of such climate resilient varieties is of great importance and therefore their quality seed need to be made available since varieties cannot be replaced until and unless seed in sufficient quantities at right time and right place at affordable price are made available to farming communities and thus necessitating for enhancing seed security for adaptation to climate change. Furthermore, ensuring the availability of quality seed of the appropriate varieties in adequate quantity at right time poses greater challenge rather than production per se.

The seed crop is affected by climate change through influencing crop phenology, reproduction, seed setting, seed-filling duration, seed size, seed dormancy, seed yield and ultimately seed quality, and hence strategies to mitigate climate change effect by approaches which affect seed production and supply chain management. Seed production of climate resilient crop/varieties at local levels is crucial and the associated indigenous knowledge of farmers with regard to environments and their choice of the kind of crop/varieties in marginal conditions could be harnessed through participatory varietal selection and seed production. The large scale adoption of a few improved varieties of a limited number of crop species has resulted in displacement of many traditional varieties and the loss of traditional knowledge associated with them are the hallmark of the formal seed sector. Moreover, the seed and planting material from formal seed systems is also protected by strong intellectual property rights (IPRs) through the complex web of multilateral trade agreements (MTAs), licenses, contracts, and patents etc, the situation is further complicated for small holders and marginal farmers of developing countries by enforcing free trade agreements (FTAs) through bilateral negotiations. Due to these and several others inherent inabilities of formal seed sector make farmers to opt age old tradition of farm saved seed and thus making necessary to improve the resiliency of local seed systems and can be characterized as the seed systems which have the capacity to absorb shocks and stress, and reorganize so as to maintain and strengthen seed security over time. The informal seed systems are 
critical for the production of a diversity of foods to ensure dietary diversity in smallholder communities. Many crop species integral to the informal seed system provide valuable macro- and micronutrients to the communities in which they are grown and consumed these seed systems contribute $60-90 \%$ of the seeds planted in many developing countries.

In relation to seed production, adjustment of crop calendar for quality seed production, the management of pollinators, post-harvest management, and seed treatment technologies, seed processing and seed storage need to be strengthened. The International Seed Treaty, also known as international treaty of plant genetic resources for food and agriculture (ITPGRFA) and administered by the food and agriculture organization of the united nations, is a comprehensive international agreement and in harmony with Convention on Biological Diversity (CBD) with the aims to ensure food security, also advocates participatory approach, the promotion of seed villages, community seed banks and protection/promotion of Farmers right. There are a number of instances where varieties in a number of crops including rice, sorghum, barley, maize, pigeon pea, lentil and groundnut etc. selected/identified/developed by farmers became very popular due to high level of adaptation, better grain quality and lack of suitable replacement, is a testimony of indigenous farmers wisdom.
Therefore, farmers participatory varietal selection and seed production enhances the resiliency of local seed systems. Moreover, participatory plant breeding and varietal selection speeds varietal development and dissemination as compared to conventional plant-breeding program. Since seed village and/or communities seed banks operate with utmost transparency, mutual trust and social responsibility of the seed producing farmers towards seed using fellow farmers. It is recognized that farmers' seed systems have made phenomenal contributions to the generation of genetic diversity in all crops. Moreover, at international level, Global Crop Diversity Trust (GCDT), the Global Partnership Initiative for Plant Breeding Capacity Building (GIPB), revised Global Plan of Action (GPA) for the Conservation and Sustainable Use of Plant Genetic Resources for Food and Agriculture (FAO) and the Nagoya Protocol (CBD) also emphasize on the strengthening of local seed systems to increase adaptation for climate change.

\section{Acknowledgements}

None.

\section{Conflict of interest}

The author declares no conflict of interest. 